PROCEEDINGS OF THE

AMERICAN MATHEMATICAL SOCIETY

Volume 133, Number 5, Pages 1357-1364

S 0002-9939(04)07642-7

Article electronically published on October 15, 2004

\title{
COMPUTING THE FREDHOLM INDEX OF TOEPLITZ OPERATORS WITH CONTINUOUS SYMBOLS
}

\author{
NATHAN S. FELDMAN AND PAUL MCGUIRE
}

(Communicated by Joseph A. Ball)

\begin{abstract}
We show how to compute the Fredholm index of a Toeplitz operator with a continuous symbol constructed from any subnormal operator with compact self-commutator. We also show that the essential spectral pictures of such Toeplitz operators can be prescribed arbitrarily.
\end{abstract}

\section{INTRODUCTION}

Let $\mathcal{H}$ denote a separable Hilbert space, $\mathcal{B}(\mathcal{H})$ the algebra of all bounded linear operators on $\mathcal{H}$ and $\mathcal{B}_{0}(\mathcal{H})$ the ideal of compact operators. An operator $S \in \mathcal{B}(\mathcal{H})$ is subnormal if $S$ has a normal extension. That is, if there is a normal operator $N$ on a Hilbert space $\mathcal{K} \supseteq \mathcal{H}$ such that $N$ leaves $\mathcal{H}$ invariant and $S=N \mid \mathcal{H}$. If, in addition, $N$ has no reducing subspaces containing $\mathcal{H}$, then $N$ is said to be the minimal normal extension of $S$, written as $N=$ mne $S$. If $\mu$ is a scalar-valued spectral measure of $N$ and $f \in L^{\infty}(\mu)$, then $f(N)$ is a well-defined normal operator given by the functional calculus for normal operators. We obtain the Toeplitz operator, $S_{f}$, with symbol $f$ constructed from the subnormal operator $S$ by compressing $f(N)$ to $\mathcal{H}$. Thus, $S_{f}=\operatorname{Pf}(N) \mid \mathcal{H}$, where $P$ denotes the orthogonal projection of $\mathcal{K}$ onto $\mathcal{H}$. We also define the normal spectrum of $S$, denoted by $\sigma_{n}(S)$, to be the spectrum of $N$, that is, $\sigma_{n}(S)=\sigma(m n e S)$. The normal spectrum of $S$ is where a continuous function $f$ needs to be defined in order to construct the Toeplitz operator $S_{f}$.

If $T \in \mathcal{B}(\mathcal{H})$, then $T$ is Fredholm if $T$ has closed range, $\operatorname{dim}[\operatorname{Ker}(T)]<\infty$ and $\operatorname{dim}\left[\operatorname{ker}\left(T^{*}\right)\right]<\infty$. If $T$ is a Fredholm operator, then the Fredholm index of $T$, denoted by $\operatorname{ind}(T)$, is $\operatorname{ind}(T)=\operatorname{dim}[\operatorname{Ker}(T)]-\operatorname{dim}\left[\operatorname{Ker}\left(T^{*}\right)\right]$. The essential spectrum of $T$, denoted by $\sigma_{e}(T)$, is the set of complex numbers $\lambda$ such that $(T-\lambda I)$ is not Fredholm.

It is well known that if $S=M_{z}$ is multiplication by $z$ on the Hardy space $H^{2}(\mathbb{D})$, and $f$ is a continuous function on the unit circle, then the Toeplitz operator $S_{f}$ is well-defined and the essential spectrum of $S_{f}$ is the continuous curve $f(\partial \mathbb{D})$. Thus $S_{f}$ is Fredholm if and only if $0 \notin f(\partial \mathbb{D})$. In this case, the Fredholm index of $S_{f}$ may be computed as a winding number, namely $i n d\left(S_{f}\right)=-n(f(\partial \mathbb{D}), 0)$, where $n(\gamma, \lambda)$ denotes the winding number of the continuous curve $\gamma$ around the complex number $\lambda \notin \gamma$. In general, if $S$ is a subnormal operator with compact self-commutator

Received by the editors October 6, 2003 and, in revised form, December 23, 2003.

2000 Mathematics Subject Classification. Primary 47B20, 47A53; Secondary 47A10.

Key words and phrases. Subnormal operator, essentially normal operator, Fredholm operator, Fredholm index.

(C)2004 American Mathematical Society Reverts to public domain 28 years from publication 
and $f$ is a continuous function on the normal spectrum of $S$, then the Toeplitz operator $S_{f}$ is well defined and the essential spectrum of $S_{f}$ was computed by Olin and Thomson [11] to be $\sigma_{e}\left(S_{f}\right)=f\left(\sigma_{e}(S)\right)$. This is a natural generalization of the result mentioned above for the unilateral shift. In this paper we show how to use winding numbers to compute the Fredholm index of $S_{f}$ for more general subnormal operators.

\section{Preliminaries}

If $K$ is a compact set in the complex plane, $\mathbb{C}$, then $C(K)$ will denote the set of all continuous complex-valued functions defined on $K$. For an operator $T$, the self-commutator of $T$ is $\left[T^{*}, T\right]=T^{*} T-T T^{*}$. Of course, an operator is normal if its self-commutator is zero. An operator whose self-commutator is compact is called essentially normal. We will use the well-known Brown-Douglas-Fillmore (BDF) Theorem 3 .

Theorem 2.1 (BDF Theorem). If $A$ and $B$ are two essentially normal operators and $\sigma_{e}(A)=\sigma_{e}(B)$ and $\operatorname{ind}(A-\lambda I)=\operatorname{ind}(B-\lambda I)$ for all $\lambda \notin \sigma_{e}(A)$, then $A$ is unitarily equivalent to a compact perturbation of $B$.

If $G$ is a bounded region in the complex plane, then $H^{2}(G)$ denotes the Hardy space over $G$. That is, all analytic functions $f$ on $G$ such that $|f|^{2}$ has a harmonic majorant. The essential boundary of $G$, denoted by $\partial_{e} G$, is the set of points $\lambda$ in the boundary of $G$ such that there exists a function $f$ in $H^{2}(G)$ that does not continue to be analytic in a neighborhood of $\lambda$.

Theorem 2.2. If $S=M_{z}$ on $H^{2}(G)$, then the following hold:

(1) $S$ is a pure subnormal operator.

(2) $\sigma_{e}(S)=\sigma_{n}(S)=\partial_{e} G$ and $\partial[c l G] \subseteq \partial_{e} G \subseteq \partial G$.

(3) The self-commutator of $S$ is trace class and $\operatorname{tr}\left[S^{*}, S\right]=\frac{1}{\pi} \operatorname{Area}(G)$.

For more on Hardy spaces see Conway [6] p. 203], and for proofs of (1) and (2) see Conway [4. For item (3) see Aleman [1].

Corollary 2.3. Let $\left\{G_{n}\right\}$ be a sequence of pairwise disjoint bounded regions such that $\bigcup_{n} G_{n}$ is bounded. Also, let $\left\{k_{n}\right\}$ be any sequence of positive integers. If $S_{n}=M_{z}$ on $H^{2}\left(G_{n}\right)$ and $T=\bigoplus_{n=1}^{\infty} S_{n}^{\left(k_{n}\right)}$, then $T$ has compact self-commutator.

Jordan regions and winding numbers: If $\gamma:[a, b] \rightarrow \mathbb{C}$ is a rectifiable continuous closed curve in the complex plane and $\lambda$ is a point not on the curve, then the winding number $n(\gamma, \lambda)=\frac{1}{2 \pi i} \int_{\gamma} \frac{1}{z-\lambda} d z$. The winding number is well known to be a homotopy invariant. If $\gamma$ is only a continuous curve, then one can approximate it by rectifiable curves and use the homotopy invariance to define the winding number of $\gamma$. Alternatively, if $\gamma:[a, b] \rightarrow \mathbb{C}$ is a continuous closed curve and, say, 0 is a point not on $\gamma$, then let $\theta:[a, b] \rightarrow \mathbb{R}$ be a continuous branch of the argument of $\gamma$. So, $\theta(t)$ is a continuous function and $\gamma(t)=|\gamma(t)| e^{i \theta(t)}$ for $t \in[a, b]$. Then $n(\gamma, 0):=\frac{1}{2 \pi}(\theta(b)-\theta(a))$. If $\Gamma$ is a finite system of closed curves $\gamma_{1}, \ldots, \gamma_{n}$, then $n(\Gamma, \lambda):=\sum_{k=1}^{n} n\left(\gamma_{k}, \lambda\right)$ for $\lambda \notin \bigcup_{k} \gamma_{k}$.

A Jordan region is a region bounded by a finite number of disjoint rectifiable Jordan curves. A Jordan region $G$ is positively oriented if each Jordan curve in the boundary of $G$ is oriented such that inside $(\partial G)=G$ and outside $(\partial G)=\mathbb{C} \backslash \operatorname{cl} G$, 
where inside $(\partial G)$ consists of those points $\lambda$ such that $n(\partial G, \lambda)=1$ and outside $(\partial G)$ consists of those points $\lambda$ such that $n(\partial G, \lambda)=0$.

Our results will be similar in nature to the classical results of Douglas (1968) and Abrahamse (1974) that determine the essential spectrum and index function for a Toeplitz operator on $H^{2}(\mathbb{D})$ or on multiply connected regions.

Theorem 2.4 (Douglas). If $S=M_{z}$ on $H^{2}(\mathbb{D})$ and if $f \in H^{\infty}(\partial \mathbb{D})+C(\partial \mathbb{D})$, then $\sigma_{e}\left(S_{f}\right)$ is the cluster set of $\hat{f}$ on the unit circle, where $\hat{f}$ is the harmonic extension of $f$ to the unit disk. Also, ind $\left(S_{f}-\lambda I\right)=-\lim _{r \rightarrow 1^{-}} n\left(\hat{f}\left(C_{r}\right), \lambda\right)$, for $\lambda \notin \sigma_{e}\left(S_{f}\right)$, where $C_{r}$ is the positively oriented circle with radius $r$ centered at the origin.

See Douglas [7, p. 169] or Nikolski [10, p. 259] for more details.

Abrahamse [2, p. 279] studied Toeplitz operators with continuous symbols on multiply connected regions bounded by Jordan curves.

Theorem 2.5 (Abrahamse). If $G$ is a positively oriented Jordan region, $f \in$ $C(\partial G)$, and $S=M_{z}$ on $H^{2}(G)$, then $\sigma_{e}\left(S_{f}\right)=f(\partial G)$ and $\operatorname{ind}\left(S_{f}-\lambda I\right)=$ $-n(f(\partial G), \lambda)$, for $\lambda \notin f(\partial G)$,

\section{MAin RESUlts}

In this section we want to compute the Fredholm index of a Toeplitz operator with a continuous symbol constructed from any pure subnormal operator with compact self-commutator. The first step of course would be to compute the essential spectrum. That was done by Olin and Thomson [11] in 1982.

Theorem 3.1 (Olin and Thomson). If $S$ is a subnormal operator with compact self-commutator and $f \in C\left(\sigma_{n}(S)\right)$, then $\sigma_{e}\left(S_{f}\right)=f\left(\sigma_{e}(S)\right)$.

We want to consider Toeplitz operators with continuous symbols but on arbitrary domains. Thus our results will be a combination of the theorems of Douglas and Abrahamse. Namely, we shall approximate an arbitrary bounded region by a multiply connected Jordan region, apply Abrahamse's result, and then take a limit.

Theorem 3.2. If $G$ is a bounded region and $S=M_{z}$ on $H^{2}(G)$ and $f \in C\left(\partial_{e} G\right)$, then $\sigma_{e}\left(S_{f}\right)=f\left(\partial_{e} G\right)$. If $\lambda \notin f\left(\partial_{e} G\right)$ and $\hat{f}$ is any continuous extension of $f$ to $c l G$, then there exists a compact set $K \subseteq$ int $(c l G)$ such that if $\Omega$ is any positively oriented Jordan region satisfying $K \subseteq \Omega \subseteq c l \Omega \subseteq \operatorname{int}(c l G)$, then

$$
\operatorname{ind}\left(S_{f}-\lambda I\right)=-n(\hat{f}(\partial \Omega), \lambda) .
$$

Here is an equivalent formulation of Theorem 3.2 that has the same feeling as Douglas's Theorem.

Corollary 3.3. If $G$ is a bounded region, $S=M_{z}$ on $H^{2}(G), f \in C\left(\partial_{e} G\right)$, $\hat{f}$ is any continuous extension of $f$ to $\mathrm{cl} G, \lambda \notin \sigma_{e}\left(S_{f}\right)$, and $\left\{\Omega_{n}\right\}$ is any sequence of positively oriented Jordan regions satisfying cl $\Omega_{n} \subseteq \Omega_{n+1}$, int $(\mathrm{cl} G)=\bigcup_{n=1}^{\infty} \Omega_{n}$, then

$$
\operatorname{ind}\left(S_{f}-\lambda I\right)=-\lim _{n \rightarrow \infty} n\left(\hat{f}\left(\partial \Omega_{n}\right), \lambda\right) .
$$

Proof of Theorem 3.2. The fact that $\sigma_{e}\left(S_{f}\right)=f\left(\partial_{e} G\right)$ follows easily from Theorems 2.2 and 3.1 Now let $\lambda \in \mathbb{C} \backslash f\left(\partial_{e} G\right)$. Let $U=\operatorname{int}(c l G)$. Since $\partial U \subseteq \partial_{e} G$ (see Theorem [2.2), it follows that $\lambda \notin f(\partial U)$. Since $\hat{f}$ is continuous on $c l G$, there 
exists a compact set $K \subseteq U$ such that $\lambda \notin \operatorname{cl}[\hat{f}(U \backslash K)]$. Let $\Omega$ be a positively oriented Jordan region satisfying $K \subseteq \Omega \subseteq U$. Let $S_{\Omega}=M_{z}$ on $H^{2}(\Omega)$, and let $L:=\operatorname{cl}\left[(U \backslash \Omega) \cup \partial_{e} G\right]$. Let $N$ be a normal operator such that $\sigma(N)=\sigma_{e}(N)=L$. Now consider $S \oplus N$ and $S_{\Omega} \oplus N$. Then both operators have essential spectrum equal to $L$, and both have the same index function, namely -1 on $\Omega \backslash L$ and 0 off of $c l G$. Hence by the Brown-Douglas-Fillmore Theorem (see Theorem 2.1), $S \oplus N$ is unitarily equivalent to a compact perturbation of $S_{\Omega} \oplus N$. Since $\hat{f}$ is continuous on $\operatorname{cl} G$, it is a uniform limit of polynomials in $z$ and $\bar{z}$. Thus it follows that $\hat{f}(S \oplus N)$ is unitarily equivalent to a compact perturbation of $\hat{f}\left(S_{\Omega} \oplus N\right)$. Hence they also have the same essential spectrum and index functions. Notice that $\lambda \notin \sigma_{e}(\hat{f}(S \oplus N))=\hat{f}(L)$. Thus we may compute the index as follows:

$$
\begin{array}{r}
\operatorname{ind}\left(S_{\hat{f}}-\lambda I\right)+\operatorname{ind}(\hat{f}(N)-\lambda I)=\operatorname{ind}\left[\left(S_{\hat{f}}-\lambda I\right) \oplus(\hat{f}(N)-\lambda I)\right]=\operatorname{ind}\left((S \oplus N)_{\hat{f}}-\lambda I\right) \\
=\operatorname{ind}\left(\left(S_{\Omega} \oplus N\right)_{\hat{f}}-\lambda I\right)=\operatorname{ind}\left[\left(\left(S_{\Omega}\right)_{\hat{f}}-\lambda I\right) \oplus(\hat{f}(N)-\lambda I)\right] \\
=\operatorname{ind}\left(\left(S_{\Omega}\right)_{\hat{f}}-\lambda I\right)+\operatorname{ind}(\hat{f}(N)-\lambda I) .
\end{array}
$$

But since $\hat{f}(N)$ is a normal operator we have $\operatorname{ind}(\hat{f}(N)-\lambda I)=0$. Thus,

$$
\operatorname{ind}\left(S_{\hat{f}}-\lambda I\right)=\operatorname{ind}\left(\left(S_{\Omega}\right)_{\hat{f}}-\lambda I\right)=-n(\hat{f}(\partial \Omega), \lambda)
$$

where the last equality holds by Theorem[2.5 Also, clearly $f=\hat{f}$ on $\sigma_{n}(S)\left(=\partial_{e} G\right)$; thus, $\operatorname{ind}\left(S_{f}-\lambda I\right)=\operatorname{ind}\left(S_{\hat{f}}-\lambda I\right)=-n(\hat{f}(\partial \Omega), \lambda)$.

We now see that Theorem 3.2 is a crucial step in computing the Fredholm index in general.

Theorem 3.4. Let $S$ be a pure subnormal operator with compact self-commutator, $f \in C\left(\sigma_{n}(S)\right), G=\sigma(S) \backslash \sigma_{e}(S)$ and $\left\{G_{n}\right\}_{n=1}^{\infty}$ the components of $G$. Assume $\hat{f}$ is any continuous extension of $f \mid \sigma_{e}(S)$ to $\sigma_{e}(S) \cup c l G$, and for each $n \geq 1$, let $a_{n} \in G_{n}$. If $\lambda \in \mathbb{C} \backslash \sigma_{e}\left(S_{f}\right)$, then there exists an integer $0<N<\infty$ and a compact set $K \subseteq \bigcup_{n=1}^{N} \operatorname{int}\left(c l G_{n}\right)$ such that if $\left\{\Omega_{n}\right\}_{n=1}^{N}$ is any finite collection of positively oriented Jordan regions satisfying $K \subseteq \bigcup_{n=1}^{N} \Omega_{n} \subseteq \bigcup_{n=1}^{N} c l \Omega_{n} \subseteq \bigcup_{n=1}^{N} \operatorname{int}\left(\operatorname{cl} G_{n}\right)$, then

$$
\operatorname{ind}\left(S_{f}-\lambda I\right)=\sum_{n=1}^{N} n\left(\hat{f}\left(\partial \Omega_{n}\right), \lambda\right) \operatorname{ind}\left(S-a_{n} I\right) .
$$

Proof. For each $n \geq 1$, let $S_{n}=M_{z}$ on $H^{2}\left(\operatorname{int}\left(\operatorname{cl} G_{n}\right)\right)$ and let $k_{n}=-i n d\left(S-a_{n} I\right)$. Consider the operator $T=\bigoplus_{n=1}^{\infty} S_{n}^{\left(k_{n}\right)}$. Let $N$ be a normal operator such that $\sigma(N)=\sigma_{e}(N)=\sigma_{e}(S)$. Then $T \oplus N$ has the same essential spectrum and index function as $S$. By Corollary 2.3, $T$ is essentially normal, and thus by the BrownDouglas-Fillmore Theory (Theorem[2.1) it follows that $T \oplus N$ is unitarily equivalent to a compact perturbation of $S$.

Notice that $\sigma_{e}(T) \subseteq \partial G \subseteq \sigma_{e}(S)$. Also, by construction, ind $(T-\lambda I)=\operatorname{ind}(S-\lambda I)$ for all $\lambda \notin \sigma_{e}(S)$. Since $f$ is a continuous function on $\sigma_{n}(S) \supseteq \sigma_{e}(S) \supseteq \sigma_{e}(T)=$ $\sigma_{n}(T)$, it is a uniform limit on $\sigma_{n}(S)$ of polynomials in $z$ and $\bar{z}$, from which it follows that $(T \oplus N)_{f}$ is a compact perturbation of $S_{f}$. Since $(T \oplus N)_{f}=T_{f} \oplus f(N)$ 
and since the normal operator $f(N)$ has a zero index function, it follows that $\operatorname{ind}\left(S_{f}-\lambda I\right)=\operatorname{ind}\left(T_{f}-\lambda I\right)$ for $\lambda \notin \sigma_{e}\left(S_{f}\right)$. Thus if $\lambda \notin \sigma_{e}\left(S_{f}\right)$, then

$$
T_{f}-\lambda I=\bigoplus_{n=1}^{\infty}\left(\left(S_{n}\right)_{f}-\lambda I\right)^{\left(k_{n}\right)}
$$

is a Fredholm operator, and thus all but finitely many terms in the direct sum are invertible. Thus, there exists a positive integer $N$ such that $\left(\left(S_{n}\right)_{f}-\lambda I\right)$ is invertible for all $n>N$. Thus, by Theorem 3.2 for each $n, 1 \leq n \leq N$, there exists a compact set $K_{n} \subseteq \operatorname{int}\left(c l G_{n}\right)$ such that $\operatorname{ind}\left(\left(S_{n}\right)_{f}-\lambda I\right)=-n\left(\hat{f}\left(\partial \Omega_{n}\right), \lambda\right)$ for any positively oriented Jordan region $\Omega_{n}$ satisfying $K_{n} \subseteq \Omega_{n} \subseteq \operatorname{cl} \Omega_{n} \subseteq \operatorname{int}\left(\operatorname{cl} G_{n}\right)$. Thus, letting $K:=\bigcup_{n=1}^{N} K_{n}$ we have that $K$ is a compact subset of $\bigcup_{n=1}^{N} \operatorname{int}\left(\operatorname{cl} G_{n}\right)$ and if $\left\{\Omega_{n}\right\}_{n=1}^{N}$ is any finite collection of positively oriented Jordan regions satisfying $K \subseteq \bigcup_{n=1}^{N} \Omega_{n} \subseteq \bigcup_{n=1}^{N} c l \Omega_{n} \subseteq \bigcup_{n=1}^{N} \operatorname{int}\left(c l G_{n}\right)$, then

$$
\begin{gathered}
\operatorname{ind}\left(S_{f}-\lambda I\right)=\operatorname{ind}\left(T_{f}-\lambda I\right)=\sum_{n=1}^{N} k_{n} \operatorname{ind}\left(\left(S_{n}\right)_{f}-\lambda I\right) \\
=-\sum_{n=1}^{N} \operatorname{ind}\left(\left(S_{n}\right)_{f}-\lambda I\right) \operatorname{ind}\left(S-a_{n} I\right)=-\sum_{n=1}^{N}(-1) n\left(\hat{f}\left(\partial \Omega_{n}\right), \lambda\right) \operatorname{ind}\left(S-a_{n} I\right) \\
=\sum_{n=1}^{N} n\left(\hat{f}\left(\partial \Omega_{n}\right), \lambda\right) \operatorname{ind}\left(S-a_{n} I\right) .
\end{gathered}
$$

We now restate Theorem 3.4 in a manner more closely resembling Douglas' Theorem (Theorem 2.4) where the index is computed as a limit of winding numbers. Notice that in view of Theorem[3.4 the sum in Corollary 3.5] is actually only a finite sum and the limit is eventually constant.

Corollary 3.5. Let $S$ be a pure subnormal operator with compact self-commutator, $f \in C\left(\sigma_{n}(S)\right), G=\sigma(S) \backslash \sigma_{e}(S)$, and $\left\{G_{n}\right\}_{n=1}^{\infty}$ the components of $G$. Assume $\hat{f}$ is any continuous extension of $f \mid \sigma_{e}(S)$ to $\sigma_{e}(S) \cup \mathrm{clG}$. For each $n \geq 1$, let $a_{n} \in G_{n}$ and also let $\left\{\Omega_{n, k}\right\}$ be a sequence of positively oriented Jordan regions satisfying $c l \Omega_{n, k} \subseteq \Omega_{n, k+1}$, and $\operatorname{int}\left(\operatorname{cl} G_{n}\right)=\bigcup_{k=1}^{\infty} \Omega_{n, k}$. If $\lambda \in \mathbb{C} \backslash \sigma_{e}\left(S_{f}\right)$, then

$$
\operatorname{ind}\left(S_{f}-\lambda I\right)=\lim _{k \rightarrow \infty} \sum_{n=1}^{\infty} n\left(\hat{f}\left(\partial \Omega_{n, k}\right), \lambda\right) \operatorname{ind}\left(S-a_{n} I\right) .
$$

The next result tells us that if $S$ has a zero index function, then every Toeplitz operator constructed from $S$ with a continuous symbol also has a zero index function. Conversely, if the index function of $S$ is non-zero at some point, then a Toeplitz operator with a one-to-one continuous symbol must also have a non-zero index at some point.

Proposition 3.6. Let $S$ be an essentially normal subnormal operator.

(a) If $\sigma(S) \neq \sigma_{e}(S)$, ind $(S-\lambda I) \neq 0$ for some $\lambda \in \mathbb{C}, f \in C\left(\sigma_{n}(S)\right)$, and $f$ is one-to-one on $\sigma_{e}(S)$, then ind $\left(S_{f}-\lambda I\right) \neq 0$ for some $\lambda \in \mathbb{C}$.

(b) If $\sigma(S)=\sigma_{e}(S)$ and $f \in C(\sigma(S))$, then ind $\left(S_{f}-\lambda I\right)=0$ for all $\lambda \notin \sigma_{e}\left(S_{f}\right)$. 
Proof. (a) Let $\pi$ be the projection from $\mathcal{B}(\mathcal{H})$ into the Calkin Algebra $\mathcal{B}(\mathcal{H}) / \mathcal{B}_{0}(\mathcal{H})$. Then $\pi(S)$ is a normal element in the Calkin algebra, and $f$ is a continuous function on $\sigma(\pi(S))$. So using the functional calculus for normal elements of the Calkin algebra we have $f(\pi(S))$ is also a normal element in the Calkin algebra, and $f(\pi(S))=\pi\left(S_{f}\right)$. Suppose that $\operatorname{ind}\left(S_{f}-\lambda I\right)=0$ for all $\lambda \notin \sigma_{e}\left(S_{f}\right)$. Then $S_{f}=N+K$ where $N$ is a normal operator and $K$ is a compact operator (see Theorem 2.1). Since $f: \sigma_{e}(S) \rightarrow \sigma_{e}(N)$ is a homeomorphism, let $f^{-1}$ be its inverse; so $f^{-1}: \sigma_{e}(N) \rightarrow \sigma_{e}(S)$. Now let $F: \sigma(N) \rightarrow \mathbb{C}$ be any continuous extension of $f^{-1}$ to $\sigma(N)$. We then have $\pi(F(N))=F(\pi(N))=f^{-1}(\pi(N))=f^{-1}(\pi(N+K))=$ $f^{-1}\left(\pi\left(S_{f}\right)\right)=f^{-1}(f(\pi(S)))=\pi(S)$. So, $\pi(F(N))=\pi(S)$. Thus, $S=F(N)+C$ where $C$ is some compact operator. Thus $S$ has the form "normal plus compact"; hence its index function is identically zero, a contradiction.

(b) If $\sigma(S)=\sigma_{e}(S)$, then $\operatorname{ind}(S-\lambda I)=0$ for every $\lambda \notin \sigma_{e}(S)$. By Theorem 3.4 it follows that $\operatorname{ind}\left(S_{f}-\lambda I\right)=0$ for all $\lambda \notin \sigma_{e}\left(S_{f}\right)$.

\section{Spectral pictures of Toeplitz operators}

In this section we show that one can arbitrarily prescribe the essential spectrum and index function of a Toeplitz operator with continuous symbol constructed from a pure subnormal operator with compact self-commutator. We also give an example showing that the spectrum of a Toeplitz operator cannot in general be computed as the essential spectrum together with those holes in the essential spectrum with non-zero index.

Theorem 4.1. Let $K$ be a non-empty compact set in the complex plane and $\left\{G_{n}\right\}$ the bounded components of $\mathbb{C} \backslash K$. If $\left\{a_{n}\right\}$ is any sequence of integers, then there exists a pure subnormal operator $S$ with compact self-commutator and a continuous function $f$ on $\sigma_{n}(S)$ such that $\sigma_{e}\left(S_{f}\right)=K$ and ind $\left(S_{f}-\lambda I\right)=a_{n}$ for $\lambda \in G_{n}$.

Recall that a "Cantor Set" is any compact metric space that is totally disconnected and perfect (has no isolated points). It is well known that any two Cantor sets in this sense are homeomorphic. We need the following well-known result (see Exercise 13, p. 285 in Conway [5]).

Lemma 4.2. If $X$ is a compact metric space and $L$ is a Cantor set, then there is a continuous function $f: L \rightarrow X$ such that $f(L)=X$.

Proof of Theorem 4.1. Case I: $\mathbb{C} \backslash K$ is connected or $a_{n}=0$ for each $n$.

Let $L$ be the classical middle-thirds Cantor set only constructed within the interval $[0,1 / 2]$. By Lemma 4.2, let $f: L \rightarrow K$ be a continuous function with $f(L)=K$. Let $S$ be an irreducible essentially normal subnormal operator with spectrum equal to the closed unit disk and essential spectrum equal to $(\partial \mathbb{D} \cup L)$ (see $[8]$ ). Choose a point $\alpha \in K$, and then extend $f$ to be defined on all of $\sigma_{e}(S)$ by defining $f$ to be constantly equal to $\alpha$ on $\partial \mathbb{D}$. Now extend this $f$ in an arbitrary manner to be continuous on all of $c l \mathbb{D}$. Then $S_{f}$ is well-defined, and $\sigma_{e}\left(S_{f}\right)=f\left(\sigma_{e}(S)\right)=f(L) \cup\{\alpha\}=K$. Now we will show that $\operatorname{ind}\left(S_{f}-\lambda I\right)=0$ for all $\lambda \in \mathbb{C} \backslash K$. It follows from Theorem 3.4 that $\operatorname{ind}\left(S_{f}-\lambda I\right)=-n(f(\partial \mathbb{D}), \lambda)$ for $\lambda \notin K$. However, since $f$ is constant on $\partial \mathbb{D}$ it follows easily that this winding number, and hence the index, is zero.

Case II: $\mathbb{C} \backslash K$ is disconnected and $a_{n} \neq 0$ for some $n$.

For convenience and without loss of generality, let us translate $K$ and assume that $K$ lies in the upper half-plane. Let $\left\{G_{n}: n \in P\right\}$ be the bounded components 
of $\mathbb{C} \backslash K$ where the corresponding integer $a_{n}$ is positive. Also let $\left\{G_{n}: n \in N\right\}$ be the bounded components of $\mathbb{C} \backslash K$ where the corresponding integer $a_{n}$ is negative. Let $A=P \cup N$. For a set $B \subseteq \mathbb{C}$, let $B^{*}=\{\bar{z}: z \in B\}$.

Let $T_{1}$ be a pure essentially normal subnormal operator such that $\sigma\left(T_{1}\right)=$ $c l \bigcup_{n \in N} G_{n}, \sigma_{e}\left(T_{1}\right)=c l \bigcup_{n \in N} \partial G_{n}$, and for each $n \in N$, ind $\left(T_{1}-\lambda I\right)=a_{n}$ for $\lambda \in G_{n}$ (see [8]). Also, let $T_{2}$ be a pure essentially normal subnormal operator such that $\sigma\left(T_{2}\right)=c l \bigcup_{n \in P} G_{n}^{*}, \sigma_{e}\left(T_{2}\right)=c l \bigcup_{n \in P} \partial G_{n}^{*}$, and for each $n \in P$, $\operatorname{ind}\left(T_{2}-\lambda I\right)=-a_{n}$ for $\lambda \in G_{n}^{*}([8])$.

Now by Case I, let $T_{3}$ be an irreducible subnormal operator with spectrum disjoint from $\sigma\left(T_{1}\right) \cup \sigma\left(T_{2}\right)$ (translate $T_{3}$ if necessary) and $f$ a continuous function on $\sigma\left(T_{3}\right)$ such that $\sigma_{e}\left(\left(T_{3}\right)_{f}\right)=f\left(\sigma_{e}\left(T_{3}\right)\right)=K$ and $\operatorname{ind}\left(\left(T_{3}\right)_{f}-\lambda I\right)=0$ for all $\lambda \notin K$. Now extend $f$ to $\sigma\left(T_{1}\right) \cup \sigma\left(T_{2}\right)$ by defining $f(z)=z$ if $z \in \sigma\left(T_{1}\right)$ and $f(z)=\bar{z}$ if $z \in \sigma\left(T_{2}\right)$. Let $S=T_{1} \oplus T_{2} \oplus T_{3}$. Then $f$ is well-defined and continuous on $\sigma(S)$. Also, $S$ is a pure essentially normal subnormal operator, and one easily checks that $\sigma_{e}\left(S_{f}\right)=f\left(\sigma_{e}(S)\right)=K$ and that $\operatorname{ind}\left(S_{f}-\lambda I\right)=a_{n}$ for $\lambda \in G_{n}$.

If $S=M_{z}$ on $H^{2}(\mathbb{D})$ and $f \in C(\partial \mathbb{D}$ ), then it is well known (see [7]) that $\sigma\left(S_{f}\right)=f(\partial \mathbb{D}) \cup\left\{\lambda: \operatorname{ind}\left(S_{f}-\lambda I\right) \neq 0\right\}$. We now give an easy example to show that in general the spectrum of a Toeplitz operator is not equal to the essential spectrum together with those points where the index is non-zero.

Example 4.3. Let $S_{1}=M_{z}$ on $H^{2}(\mathbb{D})$, let $G=\{z:|z-3|<1\}$, and let $S_{2}=M_{z}$ on $H^{2}(G)$. Let $S=S_{1} \oplus S_{2}$. If $f$ is defined on $\partial(\mathbb{D} \cup G)$ by $f(z)=z$ if $z \in \partial \mathbb{D}$ and $f(z)=\overline{z-3}$ if $z \in \partial G$, then clearly, $\sigma\left(S_{f}\right)=c l \mathbb{D}, \sigma_{e}\left(S_{f}\right)=\partial \mathbb{D}$, yet $\operatorname{ind}\left(S_{f}-\lambda I\right)=0$ for $\lambda \in \mathbb{D}$. So, $\sigma\left(S_{f}\right) \neq \sigma_{e}\left(S_{f}\right) \cup\left\{\lambda: \operatorname{ind}\left(S_{f}-\lambda I\right) \neq 0\right\}$.

It is also natural to ask if our method for computing the index (Theorem 3.4) also works for operators that are not essentially normal. The referee kindly supplied us with the following example showing that the answer is no.

Example 4.4. Let $T=M_{z}$ on $H^{2}(\Omega)$ where $\Omega=\left\{z:\left|z-\frac{1}{4}\right|<\frac{1}{4}\right\}$, and let $U$ be the unilateral shift operator of infinite multiplicity. Then consider the subnormal operator $S=T \oplus U$. Let

$$
f(z)= \begin{cases}0 & \text { for }|z|=1 \\ z & \text { for }|z|<1\end{cases}
$$

Then $S_{f}=T \oplus 0$. Thus, $\sigma(S)=\sigma_{e}(S)=\overline{\mathbb{D}}, \sigma\left(S_{f}\right)=c l \Omega$, and $\sigma_{e}\left(S_{f}\right)=\partial \Omega$. In the notation of Theorem 3.4, $G=\sigma(S) \backslash \sigma_{e}(S)=\emptyset$. Hence there are no winding numbers to compute, but $\operatorname{ind}\left(S_{f}-\lambda I\right)=\operatorname{ind}(T-\lambda I)=-1$ if $\lambda \in \Omega$.

\section{Open QUestions}

Question 5.1. If $S$ is a pure essentially normal subnormal operator and $f \in$ $C\left(\sigma_{n}(S)\right)$, then how can one compute the spectrum of $S_{f}$ ?

Question 5.2. Can we characterize the spectral pictures of Toeplitz operators with continuous symbols constructed from irreducible essentially normal subnormal operators? What if the symbol is also required to be one-to-one?

When the symbol is required to be one-to-one, the above question is related to a question about subnormal generators of $C^{*}$-algebras [9]. More precisely, in [9] it is shown that if $A$ is an irreducible essentially normal operator, then $C^{*}(A)$ has a 
subnormal generator if and only if $A$ has the same spectral picture as a Toeplitz operator $S_{f}$ where $S$ is an irreducible essentially normal subnormal operator and $f$ is continuous on $\sigma_{n}(S)$ and one-to-one on $\sigma_{e}(S)$.

Question 5.3. Is there a general method to compute the index of $S_{f}$ when $S$ is not essentially normal?

\section{REFERENCES}

[1] A. Aleman, Subnormal Operators with Compact Selfcommutator, Manuscripta Math. 91, No. 3 (1996), 353-367. MR 1416717 (97j:47036)

[2] M.B. Abrahamse, Toeplitz Operators in multiply connected regions, Amer. J. Math. 96 (1974), 261-297. MR0361891 (50:14333)

[3] L.G. Brown, R.G. Douglas and P.A. Fillmore, Unitary equivalence modulo the compact operators and extensions of $C^{*}$-algebras, Lect. Notes in Math. 345, Springer-Verlag, (1973), 58-128. MR0380478 (52:1378)

[4] J.B. Conway, Spectral Properties of Certain Operators on Hardy spaces of Planar Regions, Int. Eqns. Oper. Th. 10 (1987), 659-706. MR0904484 (88h:47040)

[5] J.B. Conway, A Course in Functional Analysis, 2nd Edition, Springer-Verlag Graduate Texts in Math. vol. 96, 1990. MR1070713 (91e:46001)

[6] J.B. Conway, The Theory of Subnormal Operators, Amer. Math. Soc., Providence, RI, 1991. MR $1112128(92 \mathrm{~h}: 47026)$

[7] R.G. Douglas, Banach Algebra Techniques in Operator Theory, 2nd Edition, Springer-Verlag Graduate Texts in Math. vol. 179, 1998. MR.1634900 (99c:47001)

[8] N.S. Feldman and P. McGuire, On the Spectral Picture of an Irreducible Subnormal Operator II, Proc. Amer. Math. Soc. 131 (2003), no. 6, 1793-1801. MR1955267

[9] P. McGuire, $C^{*}$-Algebras generated by subnormal operators, J. Funct. Anal. 79 (1988), 423445. MR0953910 (90a:47063)

[10] N. Nikolski, Operators, Functions, and Systems: An Easy Reading, Volume 1: Hardy, Hankel, and Toeplitz, Math. Surveys and Mono. 92, Amer. Math. Soc., 2002. MR1864396 (2003i:47001a)

[11] R.F. Olin and J.E. Thomson, Algebras Generated by a Subnormal Operator, Trans. Amer. Math. Soc. 271 (1982), No. 1, 299 - 311. MR0648094 (84i:47063)

Department of Mathematics, Washington and Lee University, Lexington, Virginia 24450

E-mail address: feldmanN@wlu.edu

Department of Mathematics, Bucknell University, Lewisburg, Pennsylvania 17837

E-mail address: pmcguire@bucknell.edu 